\title{
Hypertension Management in Primary Care in China: Still a Long Way to Proceed
}

Haitao Li ${ }^{*}$

School of Medicine, Shenzhen University, Shenzhen, PR China

*Corresponding author: Haitao Li, PhD, Room 311, 3/F, School Hospital, Shenzhen University, Nanhai Ave 3688, Shenzhen, Guangdong, PR China, Tel: +86 755 2672 0491; E-mail: htli1223@szu.edu.cn

Received date: February 23, 2016; Accessed date: March 02, 2016; Published date: March 10, 2016

Copyright: @ $2016 \mathrm{Li} \mathrm{H}$. This is an open-access article distributed under the terms of the Creative Commons Attribution License, which permits unrestricted use, distribution, and reproduction in any medium, provided the original author and source are credited.

\begin{abstract}
The prevalence of hypertension increased dramatically during the last several decades in China. The Chinese government has launched a national comprehensive hypertension management program in primary care, which includes hypertension detection, measurement, treatment and follow-up. The awareness, treatment and control rates of hypertension in China have improved in recent years, which is possibly attributable to the program to some extent. However, policy makers are facing many issues regarding hypertension management. This study pointed out two major issues. The first one was the low hypertension management rate. The other one was the hypertension management process itself-there were significant gaps between standards of care and medical practice. Possible barriers to effective delivery of hypertensive care were elaborated which may help improve hypertension management in primary care in China.
\end{abstract}

Keywords: Hypertension; High blood pressure; Primary care; China

\section{Abbreviations}

CVD: Cardiovascular Disease; HTN: Hypertension; BP: Blood Pressure; WHO: World Health Organization

\section{Main Text}

Cardiovascular disease (CVD) is the leading cause of death and a leading contributor to the global disease burden throughout the world. In China, CVD is estimated to account for approximately $38 \%$ of total mortality [1]. CVD events are predicted to increase by $23 \%$ by 2030 , resulting in an additional 21 million CVD events and 7.7 million deaths [2]. One of the most important risk factors of CVD is hypertension (HTN). Studies have shown a strong positive and continuous relationship between cardiovascular risk and blood pressure (BP): the risk for CVD doubles with each increment of 20 $\mathrm{mmHg}$ in systolic BP, or $10 \mathrm{mmHg}$ in diastolic BP [3]. All HTN subtypes are found to be correlated with the development of CVD among Chinese adults [4]. In 2005, an estimated 2.3 million CVD deaths and 1.3 million premature CVD deaths were attributable to HTN in China [5].

Researchers have highlighted the global need to address HTN, which is particularly urgent in low- and middle-income countries where prevalence is increasing. In China, the prevalence of HTN increased sharply from $5.1 \%$ in 1959 [6] to $7.7 \%$ in 1979 [7], and escalated from $11.9 \%$ in 1991 [8] to $34 \%$ in 2012 [9]. In 2012, the World Health Assembly set a goal of $25 \%$ reduction in the prevalence of HTN. The WHO Report 2008 has clearly re-affirmed the relevance of the primary care approach today in dealing with the increased challenges of chronic diseases. The WHO Report 2013 outlines primary care programs for any country initiative to address HTN. The New Health Care Reform Plan approved by the State Council of China in 2009 re-affirmed the key role of primary care in managing chronic illnesses including HTN [10]. Primary care, known as community health service in China, is usually provided by Community Health Centers in urban areas and township hospitals in rural areas.

The Chinese government has launched a national comprehensive HTN management program in primary care, which includes HTN detection, measurement, treatment and follow-up. According to the program, there are several approaches for the detection of HTN. Screening of health records, which are required to be established for everyone living in the community, is an approach to detect or recognize those with high risk of developing HTN. Free health examination-that includes the measurement of $\mathrm{BP}$ is another available approach for HTN detection. Hypertensive patients can also be detected through outpatient consultations. A detailed measurement protocol is provided which is the cornerstone of diagnosis and is the foundation on which management and treatment decisions are based and ultimately judged. Patients are usually classified into different management groups based on their BP levels and risk factor profiles. Lifestyle modifications and antihypertensive medications are initiated when appropriate. Specified follow-up strategies are outlined for regular monitoring of HTN. Hypertensive patients with co-morbid conditions are highlighted regarding pharmacological treatment. Different BP targets are set for patients with different age and comorbidities. Primary care providers are subject to annual inspection by local health authorities.

Even for the patients managed by primary care providers, there is still more room for improvement in the hypertensive care delivery and the followed BP control. It is shown that there are significant gaps between standards of care and medical practice in terms of lifestyle modifications and regular follow-ups. One question emerges: whether the primary care providers are ready to implement such a comprehensive HTN management program? Do they have enough resources (e.g. workforce and money) available to implement the program? Study has shown that insufficient workforce is usually complained by primary care providers, which may impair their ability 
Citation: Li H (2016) Hypertension Management in Primary Care in China: Still a Long Way to Proceed. J Gen Practice 4: 238. doi: $10.4172 / 2329-9126.1000238$

Page 2 of 2

to follow guidelines due to lack of consultation time [11-14]. Government funding for the provision of public health services including HTN management is usually not evidence-based, but largely determined by economic capacity of local governments. HTN management could be seen as adding one more thing to providers' full plate. Although computer-based electronic information system has been used to manage hypertensive patients, the segmented information system between health facilities with different levels may result in ineffective coordination. Inequalities exist in the capacity of primary care providers among different regions (i.e., between urban and rural areas; among east, middle and west part of China which are usually classified as rich, fair and poor in terms of economic status); there may be significant differences in the knowledge on the program. Different management and operation pattern of primary care facilities lead to differences in the size and financing approach etc., which also need to be considered by local health authorities when implementing the program. The essential medicine scheme has been implemented by the Chinese government aiming to facilitate affordability of and access to antihypertensive medications. The universal health insurance coverage also helps to overcome financial barriers in accessing to antihypertensive medications, especially for the poor. But, unavailability of some common used medications is often complained by patients. Antihypertensive medications provided by primary care facilities should thus be determined by both health professionals' opinions and patients' preferences. Additionally, their costeffectiveness should be considered.

\section{References}

1. (2011) World Bank. Toward a healthy and harmonious life in China: stemming the rising tide of non-communicable diseases. The World Bank Insititutes.

2. Moran A, Gu D, Zhao D, Coxson P, Chen CS, et al. (2010) Future cardiovascular disease in china: markov model and risk factor scenario projections from the coronary heart disease policy model-china. Circ Cardiovas Qual and Outcomes 3: 243-252.
3. Lewington S, Clarke R, Qizilbash N, Peto R, Collins R (2002) Age-specific relevance of usual blood pressure to vascular mortality: a meta-analysis of individual data for one million adults in 61 prospective studies. Lancet 360: 1903-1913.

4. Kelly TN, Gu D, Chen J, Huang JF, Chen JC, et al. (2008) Hypertension subtype and risk of cardiovascular disease in Chinese adults. Circulation 118: 1558-1566.

5. He J, Gu D, Chen J, Wu X, Kelly TN, et al. (2009) Premature deaths attributable to blood pressure in China: a prospective cohort study. Lancet 374: 1765-1772.

6. Huang J (1960) The summary report of the National Symposium on Heart and Vascular disease Beijing.

7. Zhou H (2004) Study on evaluation index system and method for comprehensive prevention and control community based for hypertension.

8. Wu X, Duan X, Gu D, Hao J, Tao S, et al. (1995) Prevalence of hypertension and its trends in Chinese populations. Int J Cardiol 52: 39-44.

9. Gao Y, Chen G, Tian H, Lin L, Lu J, et al. (2013) Prevalence of hypertension in china: a cross-sectional study. PLoS One 8: e65938.

10. Liu Q, Wang B, Kong Y, Cheng KK (2011) China's primary health-care reform. Lancet 377: 2064-2066.

11. Wu Y, Huxley R, Li L, Anna V, Xie G, et al. (2008) Prevalence, awareness, treatment, and control of hypertension in China: data from the China National Nutrition and Health Survey 2002. Circulation 118: 2679-2686.

12. Feng XL, Pang M, Beard J (2014) Health system strengthening and hypertension awareness, treatment and control: data from the China Health and Retirement Longitudinal Study. Bull World Health Organ 92: 29-41.

13. Li H, Wei X, Wong MC, Yang N, Wong SY, et al. (2015) A comparison of the quality of hypertension management in primary care between Shanghai and Shenzhen: a cohort study of 3196 patients. Medicine (Baltimore) 94: e455.

14. Hernandez J, Anderson S (2012) Storied experiences of nurse practitioners managing prehypertension in primary care. J Am Acad Nurse Pract 24: 89-96. 